\title{
ESTIMATIVA DO TEOR DE ARGILA E DO POTÁSSIO TROCÁVEL EM SOLOS ESPARSAMENTE AMOSTRADOS NO SUL DO ESTADO DE MATO GROSSO(1)
}

\author{
E. G. COUTO ${ }^{(2)}$, E. KLAMT $^{(3)} \&$ A. STEIN ${ }^{(4)}$
}

\begin{abstract}
RESUMO
A determinação da distribuição espacial de propriedades do solo é importante para entender as limitações para a nutrição de plantas e estabelecer o manejo apropriado do recurso solo em áreas recentemente incorporadas ao uso agrícola, princi palmente onde os levantamentos de solos disponíveis são do tipo exploratório. Como modelo de estudo, este trabal ho enfoca a variabilidade espacial do teor de argila $\left(A_{t}\right)$ e do teor de potássio trocável $\left(K_{t}\right)$ por meio de semivariogramas em amostras de solos coletadas em julho de 1994, no sul do estado de Mato Grosso. Métodos geoestatísticos foram usados para descrever a variabilidade espacial e para estimar estas propriedades ao longo de dois transectos cortando diferentes unidades de mapeamento de solos. A técnica de interpolação por Krigagem ordinária estimou apropriadamente os valores em áreas não amostradas, além de identificar os locai s onde as amostragens de solos devem-se concentrar nos futuros trabalhos de levantamentos de solos. Os atributos estudados foram influenciados principal mente pelas diferentes classes de solo e apresentaram moderada dependência espacial e anisotropia no horizonte estudado. Levantamentos para avaliar a fertilidade dos solos e informações provenientes de mapas de solos podem ser analisados, por métodos geoestatísticos, pelas instituições de planejamento e assistência técnica, para complementar informações para o planejamento do uso da terra, como também para estimar indicadores da qualidade do solo com base nos princípios da sustentabilidade das terras. Parâmetros dos semivariogramas de $A_{t}$ e $K_{t}$ associados às classes de solos podem ser usados para reduzir o esforço de amostragem destes atributos na área estudada. $O$ uso das informações dos levantamentos de solos permitiu reduzir o esforço de amostragem para a $A_{t}$, em até cerca de sete vezes.
\end{abstract}

Termos de indexação: propriedades do solo , variabilidade espacial, interpretação de dados, densidade de amostragem.

(1) Parte da Tese de Doutorado apresentada pelo primeiro autor ao Curso de Pós-Graduação em Ciência do Solo-UFRGS. Recebido para publicação em novembro de 1997 e aprovado em outubro de 1999.

(2) Professor do Departamento de Solos e Engenharia Rural, Universidade Federal do Mato Grosso. CEP 78060-900 Cuiabá (MT).

(3) Professor do Departamento de Sol os, Universidade Federal do Rio Grande do Sul, Caixa Postal 776, CEP 90001-970 Porto Alegre (RS).

(4) Professor do ITC, P.O. Box 6, 7500 AA Enschede, Holanda. 


\title{
SUMMARY: ESTIMATION OF EXCHANGEABLE POTASSIUM AND CLAY CONTENT IN SOILS WITH SCATTERED SAMPLING IN SOUTHERN MATO GROSSO, BRAZIL
}

\begin{abstract}
Thedetermination of thespatial distribution of soil properties is important to understand soil constraints to plant nutrition and appropriatemanagement of soil resources in recently cul tivated areas, particularly where only data from exploratory soil surveys areavailable. This study addresses spatial variability of clay content $\left(A_{t}\right)$ and exchangeable potassium $\left(\mathrm{K}_{\mathrm{t}}\right)$ in soil samples collected in J uly of 1994, located in Southern Mato Grosso, Brazil. Geostatistical methods were used to describe the spatial variability and predict these soil properties al ong two transects, crossing different soil mapping units and land usetypes. By ordinary kriging, values in unsampl ed areas wereappropriate y estimated and places where more intensive sampling is required were identified. Both soil properties studied were influenced mainly by different soil cl ass and exhibited a moderatespatial dependence and anisotropy in the horizon studied. Surveys of soil fertility, plus data gathered from soil survey, can beanal yzed by geostatistical methods by decision-makers, to support management planning and to predict indicators related to soil quality as a measure of sustainability. Parameters from semivariograms associated to soil dasses can be used to reduce sampling efforts to estimate $A_{t}$ and $K_{t}$ distribution and patterns in the area studied. Soil survey information allowed to reduce the sampling effort for the $A_{t}$ about seven times.
\end{abstract}

Index terms: soil properties, spatial variability, interpolation of data, soil sampling density.

\section{NTRODUÇÃO}

Os estudos de sol os realizados no estado de Mato Grosso revelam que as reservas de potássio trocável $\left(K_{t}\right)$, como de outros el ementos, são baixas, e deficiências deste nutriente têm sido reportadas por vários autores (Lopes \& Cox ,1977; Goedert, 1983; Couto et al., 1990; Couto, 1997). O teor deargila $\left(A_{t}\right)$, por sua vez, é de grande utilidade para se planejar o uso agrícola dos solos, porque em geral se correlaciona com a disponibilidade dos nutrientes e propensão à erosão (Sel by, 1993).

O sul do estado de Mato Grosso, como na maioria dos estados brasilei ros, dispõe de informações de sol o apenas referentes a levantamentos dereconhecimento ou exploratórios, como foram as produzidas pelo projeto RADAMBRASIL (RADAMBRASI L, 1982a,b). Os dados sobre solos nestes estudos restringem-se aos perfis modais, descritos e analisados para as principais classes de solos que ocorrem na região estudada. Tais perfis, como demonstrado por van der Berg \& Klamt (1997), nem sempresãorepresentativos dos sol os del ineados ecaracterizados nos levantamentos de solos. Como a execução de levantamentos de solos compatíveis para o planejamento de uso de bacias hidrográficas e propriedades agrícolas exige muito tempo eéoneroso, freqüentemente, os planejadores, extensionistas emesmo os proprietários rurais precisam de usar técnicas alternativas para a obtenção dos dados de que necessitam.

A geoestatística tem sido de grande utilidade no estudo de variações espaciais de propriedades de solos, para os mais diversos propósitos (Bouma et al., 1989; Fromm et al., 1993; M oglen \& Bras, 1995; Schlesinger et al., 1996). Os procedimentos mais comuns incluem a caracterização e o modelamento da variabilidade espacial por meio de estimativa dos valores nos locais não visitados, simulações espaciais e otimização dos esquemas de amostragens (Cambardella et al., 1994).

A variabilidade espacial dos solos pode ser dividida em variabilidade ao acaso e sistemática. A distinção entre elas depende, comumente da escala. Por exemplo, a classificação de solos baseada em atributos diagnósticos do subsolo pode ter variabilidade sistemática num levantamento de pequena escala, mas pode ter variação ao acaso em levantamentos mais detalhados. A variação sistemática é medida pelos levantamentos de solos eoutras investigações pedológicas. Perrier \& Wilding (1986) referem-se a ela como função das superfícies geomórficas e dos el ementos da paisagem, da atuação dos fatores de formação dos solos e das interações desses fatores.

Quando a variabilidade não pode ser relacionada com uma causa específica, ela é denominada de variabilidade ao acaso. Em geral, espera-se que a variabilidade sistemática de sol os não modificados pelo uso deva ser maior do que a variabilidade ao acaso. Entretanto, a relação entre a variação sistemática eao acaso depende da escala consi derada (Upchurch \& Edmonds, 1991).

Os estudos da variabilidade espacial e da interpolação de dados de sol os têm sido feitos para distâncias, variando de centímetros a centenas de metros (Reichardt et al., 1986; Cambardella et al., 
1994; Souza et al., 1997). Entretanto, há poucas aplicações destes métodos para distâncias de quilômetros (Yost et al., 1982a, b; Y ost et al., 1982b; Uehara et al., 1985; van der Berg \& Klamt, 1997), o que pode ser útil na obtenção de dados para planejamento de uso de solos e mesmo para execução de levantamentos detal hados (Voltz et al., 1997).

Estes estudos podem estimar, numa escala regional, a precisão dos mapas de solos a partir da caracterização e interpretação das estruturas espaciais de propriedades do solo, determinadas usualmente em laboratórios de rotina. Neste contexto, o presente trabal ho objetivou identificar, numa escal a regional, a variabilidade espacial doteor de argila e do potássio trocável associada às classes de solos e aos principais usos da terra, bem como relacionar tal variabilidade espacial com o uso atual.

\section{MATE RIAL E MÉTODOS}

A área de estudo está local izada no sul do estado de Mato Grosso, Centro-Oeste do Brasil (Figura 1), compreendendo cerca de $10.000 \mathrm{~km}^{2}$. A área é caracterizada pela vegetação de Cerrado e suas tipificações (RADAMBRASI L, 1982a,b). A dassificação climática da região é do tipo Aw com precipitação média anual de $1.557 \mathrm{~mm}$, com o período chuvoso de setembro/outubro a abril/maio. A temperatura média anual é de $23,3 \circ C$, observando-se um declínio pouco sensível nos meses de junho a agosto (Campelo J r. et al., 1990).
Como representado na figura 2, os principais sol os na parte norte e sul da área são, respectivamente, Latossolos Vermel ho-Amarelos distróficos em rel evo plano no topo das superfícies e Podzól icos Vermel hoAmarelos eutróficos normalmente localizados nas áreas de relevo com forma aguçada e convexa. Nas partes mais baixas da paisagem, com relevo tabuliforme, dominam as Areias Quartzosas álicas com subdominância de solos Concrecionários. $\mathrm{Na}$ parte leste, ocorrem numa proporção semel hanteos Podzól icos Vermel ho-Amarel os eutróficos nas áreas de relevo com forma aguçada e convexa, e as Areias Quartzosas álicas nas partes mais baixas da paisagem, com rel evotabuliforme (RADAMBRASI L, 1982a,b).

Os tipos de usos da terra foram defini dos por meio da interpretação da composição col orida das bandas 2,3 e 4 de imagens Landsat-TM, enquanto as unidades fisiográficas foram determinadas por meio das cartas topográficas do DSG/I BGE na escala de 1:100.000, e dos mapas exploratórios de solos, geol ógi co e geomorfológico, na escala de 1:1.000.000 (RADAMBRASIL, 1982a,b). As amostras foram coletadas ao acaso nas diferentes unidades fisiográficas e classes de solos e principais usos da terra, perfazendo um total de 67 amostras, para o horizonte A, e 47, para o horizonte B (enfatizou-sea coleta dos horizontes superficiais, os mais influenciados pelo uso; desta forma, cerca de 20 horizontes subsuperficias, principalmente de Areias Quartzosas, não foram coletados). O georeferenciamento das amostras foi feito por meio de um GPS Trimble com precisão de 25 metros (Trimble, 1993).

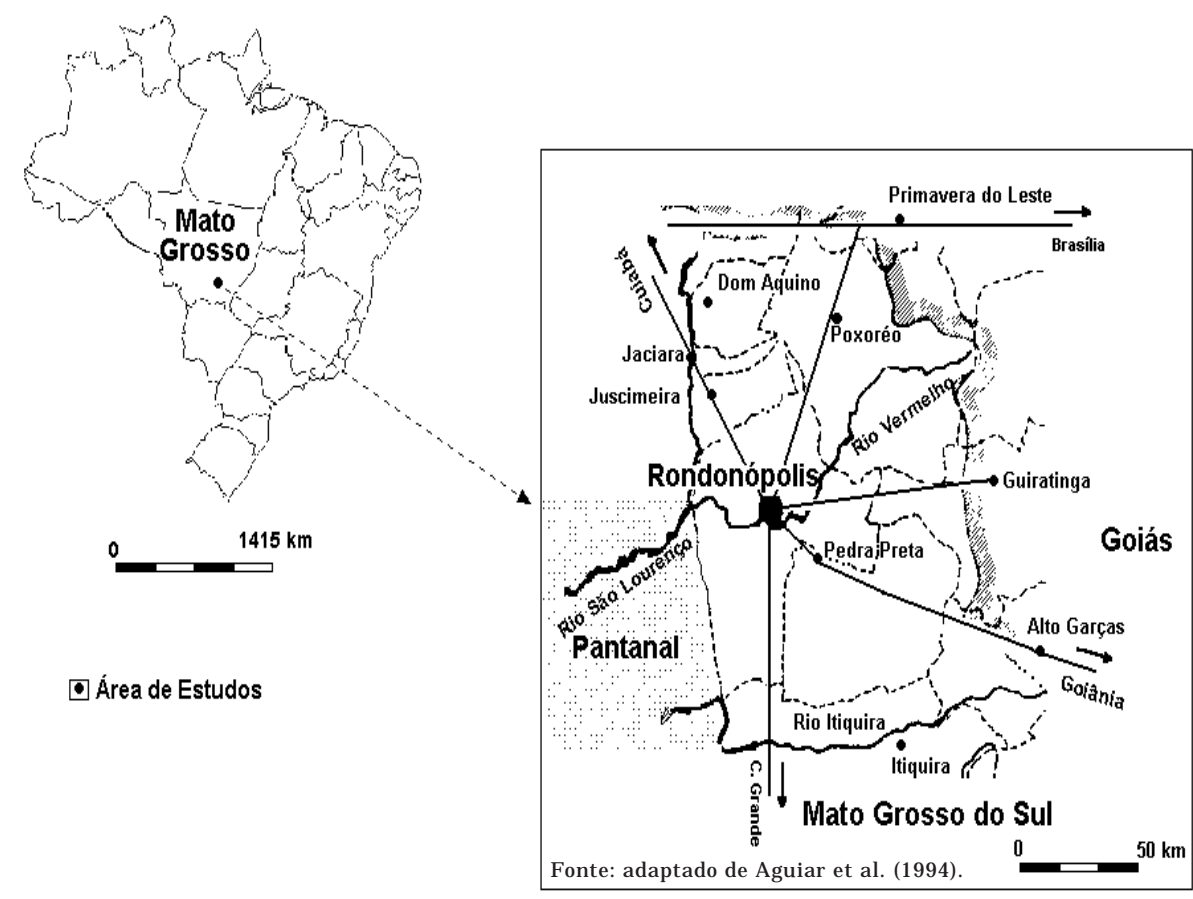

Figura 1. Localização da área de estudos. 


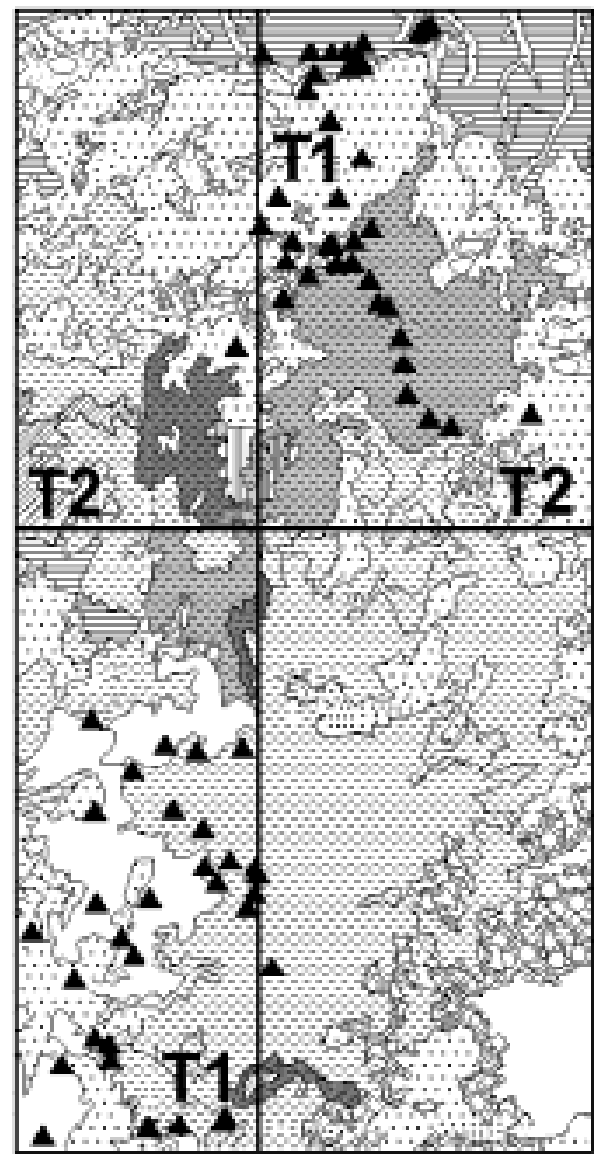

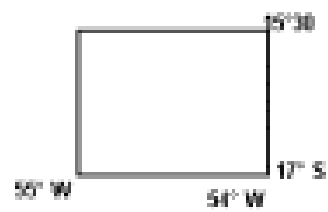

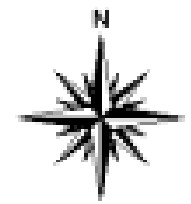

Unidade de Mapeamento

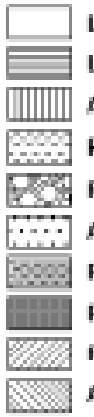

LEa] LE álicos

LWes LV distróficos

A0 a1 $A Q$ álicas

PE 13 PV eutróficos

Ret Lit. eutróficos

A0a $A Q$ álicas

PEa PV eutróficos

PE 12 PV eutróficos

PE PV eutróficos

A0\% AQ álicas

Use mais frequente

culturas anuais

culturas anuais $=$ cerrado

pastagens cultivadas bem manejadas

pastagens cultivadas bem manejadas

floresta subcaducifólia

cerrado

pastagens cultivadas bem manejadas

pastagens cultivadas bem manejadas

pastagens cultivadas bem manejadas

cerrado + pastagens
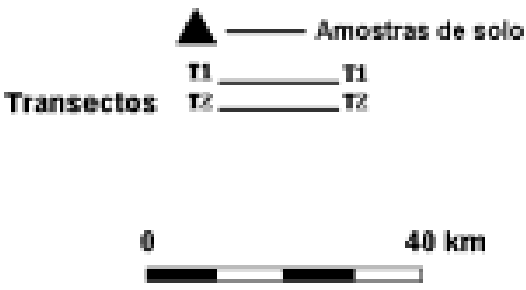

Figura 2. Mapa generalizado de solos e usos da terra mais freqüentes na área de estudos, incluindo os transectos imagi nários $\mathrm{T}_{1}$ e $\mathrm{T}_{2}$ (adaptados de RADAMBRASI L, 1982a,b).

As amostras dos horizontes for am col etadas por meio de tradagem e analisadas de acordo com os procedimentos da EMBRAPA (1997). Os atributos usados neste trabal ho, teor de argila $\left(A_{t}\right)$ e potássio trocável o $\left(\mathrm{K}_{\mathrm{t}}\right)$, foram determinados, respectivamente, pelo densímetro de Bouyoucos após dispersão com $\mathrm{NaOH}$ epor fotometria de chama a partir da extração em solução Mehlich-1 $\left(\mathrm{HCl} 0,05 \mathrm{M}+\mathrm{H}_{2} \mathrm{SO}_{4}\right.$ $0,0125 \mathrm{M})$.

\section{Procedimentos estatísticos}

A análise estatística foi realizada em duas etapas. Primeiramente, utilizou-se a estatística clássica incluindo a média, mínimo, máximo, amplitude, desvio-padrão e coeficiente de variação. Em segundo lugar, aplicou-se o teste de médias (teste de Tukey a $5 \%)$ para identificar os efeitos do uso da terra nas propriedades dos solos estudadas, avaliar as diferenças entre horizontes e entre classes de sol os. As análises estatísticas foram realizadas por meio dos programas Systat (Wilkinson, 1992), e SPSS (Spss, 1994).
E mbora estes métodos estatísticos permitam inferir sobre a variabilidade do solo (vertical e horizontal ), a dependência espacial entreas amostras somente pode ser modelada por meio da geoestatística (Webster, 1985), utilizando o semivariograma, a partir do número total de observações (67 amostras, para o horizonte A, e 47 amostras, para o horizonte B), conforme se pode visualizar na figura 2. Os semivariogramas, a partir das pressuposições de estacionariedade da hipótese intrínseca, foram estimados a partir da seguinte equação:

$$
\left.\gamma *(\mathrm{~h})=\frac{1}{2 \mathrm{~N}_{\mathrm{i}}(\mathrm{h})} \sum_{\mathrm{j}=1}^{\mathrm{N}_{\mathrm{i}}(\mathrm{h})}\left[\mathrm{z}_{\mathrm{i}}\right)-\mathrm{z}\left(\mathrm{s}_{\mathrm{i}}+\mathrm{h}\right)\right]^{2}
$$

em que $N(h)$ é o número de pares de pontos $z\left(s_{i}\right)$ e $z\left(s_{i}+h\right)$, separados por uma distância $h$, informando quão diferentes tornam-se os valores em função de h. N ormal mente, o semivariograma é representado pel o gráfico $\gamma^{*}(\mathrm{~h})$ versus $\mathrm{h}$. Do ajuste de um model o matemático aos valores calculados de $\gamma^{*}(\mathrm{~h})$ são 
definidos os coeficientes do modelo teórico para o semivariograma (o efeito pepita, $\mathrm{C}_{0}$; o patamar, C; e o al cance de dependência espacial, a). O efeito pepita é o valor da semivariância para a distância zero e representa o componente da variação ao acaso; o patamar é o valor da semivariância em que a curva estabiliza sobre um valor constante; o alcance é a distância da origem até onde o patamar atinge valores estáveis, expressando a distância além da qual as amostras não são correlacionadas (Trangmar et al., 1985). Neste estudo, este tipo de semivariograma foi definido como sendo "total".

Determinou-se, também, o semivariograma denominado "intra-estratos" (within-strata semivariogram), um conceito recentementeintroduzido (Stein, 1998), no qual o semivariograma é estimado, tomando-se em consideração todos os pares de pontos que caem dentro do mesmo estrato (que consistenos delineamentos de solo ou de uso da terra). Tal semivariograma permite avaliar se o uso da estratificação, quando comparado à ausência da mesma, reduz o número de observações necessárias para determinar os coeficientes do modelo geoestatístico (efeito pepita, patamar e alcance).

A interpolação foi realizada por meio de krigagem, estimando-se os val ores das propriedades do sol o para locais não amostrados. A estimativa dos valores foi realizada por meio da seguinte equação:

$$
\mathrm{Z}^{*}\left(\mathrm{x}_{0}\right)=\sum_{\mathrm{i}=1}^{\mathrm{n}} \lambda_{\mathrm{i}} \mathrm{Z}\left(\mathrm{x}_{\mathrm{i}}\right)
$$

em que: $\lambda$ é ponderador, e $Z(x)$, o valor observado. Neste estudo, a técnica da krigagem foi aplicada para estimar o teor de argila $\left(A_{t}\right)$ e do potássio trocável $\left(K_{t}\right)$ ao longo das unidades de mapeamento de sol os delineadas a partir de um mapa exploratório (RADAMBRASIL, 1982a,b). Para avaliar o comportamento destas propriedades ao longo de grandes distâncias, construíram-se os transectos imaginários 1 e $2\left(T_{1} \mathrm{e} T_{2}\right)$, visual izados na figura 2 , os quais cortavam unidades de uso da terra e, ou, unidades de solos previamente identificadas nos trabal hos de campo, tendo sido traçados a partir do ponto médio na direção leste-oeste e norte-sul da área representada pelos pontos amostrados (Couto et al., 1997). Nestes transectos, estimaram-se, por meio de krigagem ordinária, os valores de $K_{e}$ e $A_{t}$ para o horizonteA em interval os regulares de $1,0 \mathrm{~km}$, a partir dos semivariogramas "totais".

Para a determinação dos semivariogramas e da krigagem, foram usados o software GS+ (Robertson, 1998) e os programas computacionais desenvol vidos por Stein \& Staritsky (1993). Além disso, os parâmetros dos semivariogramas foram usados no programa computacional OPTIM, desenvolvido por Stein (1996), o qual determina o melhor intervalo de amostragem das variáveis estudadas, para se atingir determinado nível de precisão. Estenível de precisão é expresso na mesma unidade destas variáveis, sendo definido como o valor mais el evado de incerteza que se tem num mapa interpolado. Conforme a descrição de Winkels \& Stein (1997), este valor éigual ao valor mais el evado do desvio-padrão da krigagem, correspondendo ao valor estimado no centro de uma célula de grade quadrada, para observações realizadas nos vértices da referida célula. O desvio-padrão da krigagem éexpresso pela seguinte fórmula:

$$
\text { Desvio - padrão da krigagem }=\sqrt{g_{0}^{\prime} G^{-1} g_{0}-x_{a} V x_{a}}
$$

em que $g_{0}$ éo vetor com os val ores do semivariograma entre o local observado e o local estimado, G é a matriz $n \times n$ com os valores do semivariograma entre os locais observados, In é o vetor de $n$ elementos , todos iguais a 1, sendo:

$$
\mathrm{x}_{\mathrm{a}}=1-\mathrm{g}^{\prime} \mathrm{G}^{-1} 1_{\mathrm{n}} \quad \text { e } \quad \mathrm{V}=\left(1^{\prime} \mathrm{n}^{-1} 1_{\mathrm{n}}\right)^{-1}
$$

Considerando que a equação que expressa o desvio-padrão não depende do vetor das observações, uma vez que isto depende do variograma e da configuração que os dados apresentam, é possível utilizá-la para otimizar o esquema de amostragem. Neste estudo, o programa interativo OPTIM foi usado para calcular o menor espaçamento entre os pontos que permitissem identificar o nível crítico do potássio para os solos da região (cerca de $0,15 \mathrm{cmol}_{\mathrm{c}} \mathrm{kg}^{-1}$, segundo Lopes \& Cox, 1977) e separar a classe das Areias Quartzosas das outras classes (150 $\mathrm{g} \mathrm{kg}^{-1}$ de argila) sobre um mapa interpolado.

\section{RESULTADOSE DISCUSSÃO}

\section{Variabilidade das propriedades selecionadas a partir dos valores médios}

A média dos valores do potássio trocável $\left(K_{t}\right)$ do horizonte $A$ foi significativamente maior do que no horizonte B (Quadro1). O val or mais el evado de $K_{t}$ no horizonte $A$ das áreas com pastagens cultivadas deve estar relacionado com a mobilização deste el emento, pela queima periódica durante a estação seca, uma prática comum nestas áreas (Coutinho,1990), e com o fato de situar-se grande parte destas áreas em Podzólicos Vermel ho-Amarel os eutróficos.

Nas classes de solos, os Podzólicos VermelhoAmarelos eutróficos e os Latossolos VermelhoEscuros apresentaram as médias mais el evadas no horizonte superficial, eas Areias Quartzosas, as mais baixas para os horizontes subsuperficiais. Esta distribuição é explicada pelas características das próprias classes de solos, mas pode também estar relacionada com as queimadas periódicas, calagem e adubação por meio de operações mecanizadas. 
Quadro 1. Sumário da estatística descritiva para o teor de potássio trocável e para o teor de argila nos horizontes A e B, associados ao uso da terra e classes de solo

\begin{tabular}{|c|c|c|c|c|c|c|c|}
\hline & Horizonte & $\mathbf{N}$ & Média(1) & Sd & cV & Mínimo & Máximo \\
\hline \multicolumn{8}{|l|}{ Potássio (cmolc kg-1) } \\
\hline Total & $\begin{array}{l}A \\
B\end{array}$ & $\begin{array}{l}67 \\
47\end{array}$ & $\begin{array}{l}0,18 \mathrm{a} \\
0,07 \mathrm{~b}\end{array}$ & $\begin{array}{l}0,15 \\
0,09\end{array}$ & $\begin{array}{l}0,86 \\
1,15\end{array}$ & $\begin{array}{l}0,02 \\
0,01\end{array}$ & $\begin{array}{l}0,51 \\
0,33\end{array}$ \\
\hline $\begin{array}{l}\text { Cultura anual } \\
\text { Pastagens cultivadas } \\
\text { Vegetação original }\end{array}$ & $A$ & $\begin{array}{l}14 \\
34 \\
19\end{array}$ & $\begin{array}{l}0,15 \mathrm{ac} \\
0,22 \mathrm{a} \\
0,11 \mathrm{bc}\end{array}$ & $\begin{array}{l}0,15 \\
0,16 \\
0,10\end{array}$ & $\begin{array}{l}0,98 \\
0,72 \\
0,94\end{array}$ & $\begin{array}{l}0,03 \\
0,03 \\
0,02\end{array}$ & $\begin{array}{l}0,51 \\
0,51 \\
0,37\end{array}$ \\
\hline $\begin{array}{l}\text { Culturas anuais } \\
\text { Pastagens cultivadas } \\
\text { Vegetação original } \\
\text { Areias Quartzosas } \\
\text { Latossol os Vermel ho-E scuros } \\
\text { Latossol os Vermel ho-Amarel os } \\
\text { Podzólicos Vermel ho-Amarel os eutróficos } \\
\text { Podzólicos Vermel ho-Amarel os distróficos } \\
\text { Areias Quartzosas } \\
\text { Latossol os Vermel ho-Escuros } \\
\text { Latossol os Vermel ho-Amarel os } \\
\text { Podzólicos Vermel ho-Amarel os eutróficos } \\
\text { Podzólicos Vermel ho-Amarel os distróficos }\end{array}$ & B & $\begin{array}{r}8 \\
27 \\
12 \\
25 \\
14 \\
14 \\
3 \\
11 \\
15 \\
11 \\
9 \\
3 \\
9\end{array}$ & $\begin{array}{l}0,06 \mathrm{ac} \\
0,10 \mathrm{a} \\
0,03 \mathrm{bc} \\
0,14 \mathrm{a} \\
0,27 \mathrm{bc} \\
0,09 \mathrm{a} \\
0,26 \mathrm{ac} \\
0,23 \mathrm{ac} \\
0,03 \mathrm{a} \\
0,07 \mathrm{ac} \\
0,04 \mathrm{a} \\
0,16 \mathrm{ad} \\
0,14 \mathrm{bcd}\end{array}$ & $\begin{array}{l}0,10 \\
0,09 \\
0,02 \\
0,13 \\
0,19 \\
0,09 \\
0,16 \\
0,14 \\
0,03 \\
0,09 \\
0,03 \\
0,12 \\
0,11\end{array}$ & $\begin{array}{l}1,66 \\
0,92 \\
0,82 \\
0,95 \\
0,69 \\
0,92 \\
0,61 \\
0,61 \\
0,86 \\
1,26 \\
0,80 \\
0,77 \\
0,74\end{array}$ & $\begin{array}{l}0,01 \\
0,01 \\
0,01 \\
0,02 \\
0,03 \\
0,03 \\
0,08 \\
0,03 \\
0,01 \\
0,02 \\
0,01 \\
0,04 \\
0,05\end{array}$ & $\begin{array}{l}0,31 \\
0,33 \\
0,08 \\
0,51 \\
0,51 \\
0,36 \\
0,38 \\
0,51 \\
0,10 \\
0,33 \\
0,11 \\
0,29 \\
0,31\end{array}$ \\
\hline \multicolumn{8}{|l|}{ Argila $\left(g_{\text { kg-1) }}\right.$} \\
\hline Total & $\begin{array}{l}\text { A } \\
\text { B }\end{array}$ & $\begin{array}{l}67 \\
47\end{array}$ & $\begin{array}{l}232,07 \text { a } \\
270,93 \text { a }\end{array}$ & $\begin{array}{l}174,39 \\
206,09\end{array}$ & $\begin{array}{l}0,75 \\
0,76\end{array}$ & $\begin{array}{l}10,0 \\
10,0\end{array}$ & $\begin{array}{l}680,0 \\
710,0\end{array}$ \\
\hline $\begin{array}{l}\text { Cultura anual } \\
\text { Pastagens cultivadas } \\
\text { Vegetação original }\end{array}$ & A & $\begin{array}{l}14 \\
34 \\
19\end{array}$ & $\begin{array}{r}276,43 \mathrm{a} \\
296,30 \mathrm{a} \\
93,53 \mathrm{~b}\end{array}$ & $\begin{array}{l}166,90 \\
145,42 \\
149,25\end{array}$ & $\begin{array}{l}0,60 \\
0,49 \\
1,60\end{array}$ & $\begin{array}{l}10,00 \\
40,00 \\
10,0\end{array}$ & $\begin{array}{l}680,0 \\
510,0 \\
610,0\end{array}$ \\
\hline Cultura anual & B & 8 & 301,25 a & 195,63 & 0,65 & 10,00 & 510,00 \\
\hline Pastagens cultivadas & & 27 & 364,78 a & 178,86 & 0,49 & 110,0 & 710,00 \\
\hline Vegetação original & & 12 & $70,83 \mathrm{~b}$ & 105,27 & 1,49 & 10,00 & 380,00 \\
\hline Areias Quartzosas & A & 25 & $44,71 \mathrm{a}$ & 35,02 & 0,78 & 10,0 & 110,0 \\
\hline Latossolos Vermel ho-Escuros & & 14 & $334,29 \mathrm{~b}$ & 167,09 & 0,50 & 110,0 & 680,0 \\
\hline Latossol os Vermel ho-Amarel os & & 14 & $307,14 \mathrm{~b}$ & 127,37 & 0,41 & 140,0 & 610,0 \\
\hline Podzólicos Vermelho-Amarel os eutróficos & & 3 & $316,67 \mathrm{~b}$ & 60,27 & 0,19 & 260,00 & 380,00 \\
\hline Podzólicos Vermel ho-Amarelos distróficos & & 11 & $470,00 \mathrm{~b}$ & 171,63 & 0,66 & 40,00 & 510,00 \\
\hline Areias Quartzosas & B & 15 & $57,50 a$ & 48,37 & 0,84 & 10,00 & 140,00 \\
\hline Latossol os Vermel ho-E scuros & & 11 & $353,00 \mathrm{~b}$ & 121,11 & 0,34 & 180,00 & 510,00 \\
\hline Latossol os Vermel ho-Amarel os & & 9 & $362,86 \mathrm{~b}$ & 145,45 & 0,40 & 140,00 & 510,00 \\
\hline Podzólicos Vermelho-Amarel os eutróficos & & 3 & $296,67 \mathrm{~b}$ & 75,06 & 0,25 & 210,00 & 340,00 \\
\hline Podzólicos Vermel ho-Amarelos distróficos & & 9 & $538,57 \mathrm{c}$ & 141,35 & 0,26 & 340,00 & 710,00 \\
\hline
\end{tabular}

(1) Médias seguidas da mesma letra, na coluna, não diferem entre si pelo teste de Tukey a 5\%.

Também o histórico de uso podeter contribuído para aumentar as concentrações desse elemento, principalmente nos solos intensamente cultivados como os Latossol os Vermel ho-E scuros (Couto et al., 1990; Couto, 1997).

Os Podzól i cos Vermel ho-Amarelos eutróficos, que são os sol os mais bem providos de nutrientes na área de estudos, mostraram os mais el evados val ores de $\mathrm{K}_{\mathrm{t}}$ nos dois horizontes. Os Latossolos VermelhoEscuros e Vermelho-Amarelos, ambos muito similares em seus atributos químicos efísi cos quando sob vegetação original (RADAMBRASIL, 1982a,b; Oliveira et al., 1992) são muito distintos nos usos mais intensivos. Os mai ores valores de $\mathrm{K}_{\mathrm{t}}$, associados aos Latossolos Vermelho-Escuros, refletem o uso mais intensivo desta classe, principalmente com a cultura da soja, confirmando os resultados obtidos em trabal hos na mesma região (Couto et al., 1990), onde os autores mostraram que o uso agrícol a nesta área tem modificado profundamente as propriedades químicas destes solos. 
As médias dos teores de argila $\left(A_{t}\right)$ aumentaram com a profundidade, o que era de se esperar, principalmente por se tratar de área com grande ocorrência de Podzólicos. Quando se consideraram os diferentes usos da terra, as áreas com pastagens cultivadas apresentaram as maiores médias de valores de $A_{t}$, para ambos os horizontes (Quadro 1). A análise estatística mostrou haver diferença significativa entre as áreas com uso agrícola (agricultura ou pecuária) e as sob vegetação original, o que é devido às diferentes classes de solos embutidas nos usos. Isto confirma a observação efetuada no campo de que apenas os sol os arenosos ainda estavam sob vegetação original. Os mais baixos valores, como era esperado, foram observados na classe das Areias Quartzosas, enquanto os demais apresentaram teores consistentes com as classes a que pertencem, confirmando os resultados apresentados nos levantamentos exploratórios previamenterealizados (RADAMBRASI L, 1982a,b).

\section{Dependência espacial das propriedades selecionadas}

O variograma total do $K_{t}$ seguiu um modelo esférico e apresentou dependência espacial para ambos os horizontes (Figura 3a,b). Os alcances da dependência espacial foram, respectivamente, de 34,9 e 12,9 km, para os horizontes A eB. Os efeitos pepitas foram, respectivamente, de 0,0119 $\left(\mathrm{cmol}_{\mathrm{C}} \mathrm{kg}^{-1}\right)^{2} \mathrm{e}$ $0,0002\left(\mathrm{cmol}_{\mathrm{C}} \mathrm{kg}^{-1}\right)^{2}$, para os horizontes $\mathrm{A}$ e $\mathrm{B}$, isto significa dizer que o efeito pepita do horizonte $B$ foi cerca de sessenta vezes mais baixo do que no horizonte A. Diferenças nas práticas de manejo adotadas pelos agricultores, incluindo o tipo e a quantidade de fertilizantes incorporados no solo, influíram nas propriedades químicas no horizonte $A$, produzindo um efeito pepita muito mais el evado neste horizonte.

A mesma tendência foi observada para o variograma exponencial de $A_{t}$ (Figura $\left.3 c, d\right)$, em que o efeito pepita do horizonte A $\left(7.490\left(\mathrm{~g} \mathrm{~kg}^{-1}\right)^{2}\right)$ foi cerca de 3,5 vezes superior ao do horizonte B (2.200 $\left.\left(\mathrm{g} \mathrm{kg}^{-1}\right)^{2}\right)$, indicando haver grande número de classes de solos com textura superficial bem distinta, em distâncias relativamente curtas, refletindo diferentes materiais de origem, propiciando maior fonte de variação espacial.

Os variogramas de $K_{t}$ e $A_{t}$, em ambos os horizontes, são distintos nas diferentes direções (Quadro 2), mostrando estruturas de dependência variadas. Istoé, o $\mathrm{K}_{\mathrm{t}}$ e $\mathrm{A}_{\mathrm{t}}$ variam anisotropicamente, como se pode observar pelas diferenças nas razões dos alcances dos variogramas individuais para as direções indicadas (Trangmar et al., 1985). A direção da máxima variação para ambas as propriedades baseada neste critério (razão entre o alcance sulnorte e leste-oeste) foi a direção sul-norte, para o horizonte A (Quadro 2). Os valores da razão anisotrópica foram, respectivamente, de 2,40 para o teor de argila $\left(A_{t}\right)$ e 2,15 para o potássio trocável $\left(\mathrm{K}_{\mathrm{t}}\right)$, o que está dentro da magnitude dos valores encontrados para a maioria das propriedades do solo (a) $\mathrm{K}-\mathrm{HzA}$

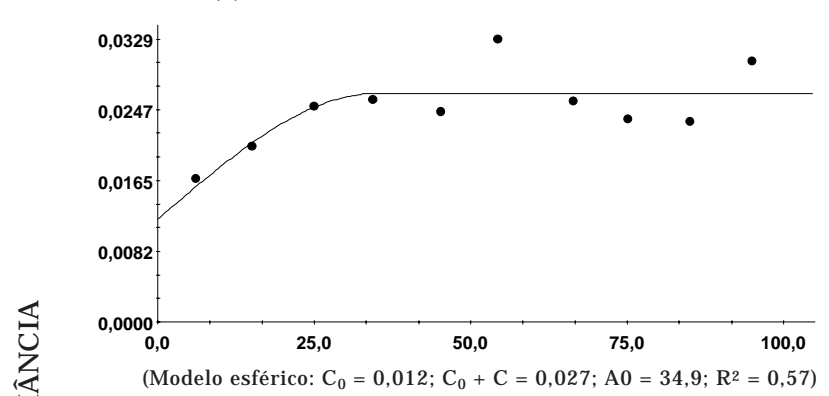

(c) Argila - Hz A

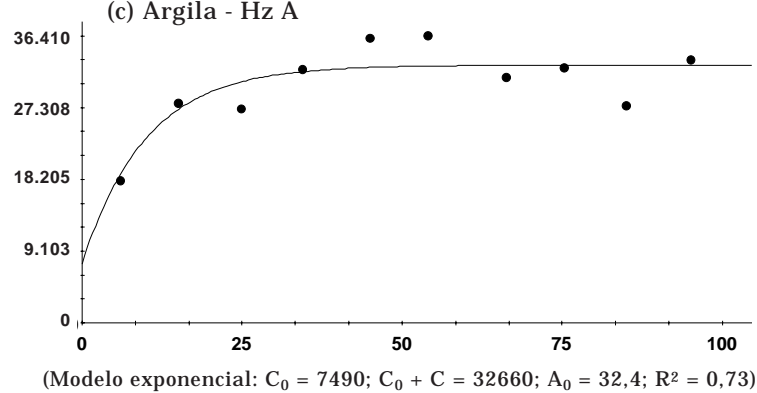

(b) $\mathrm{K}-\mathrm{HzB}$
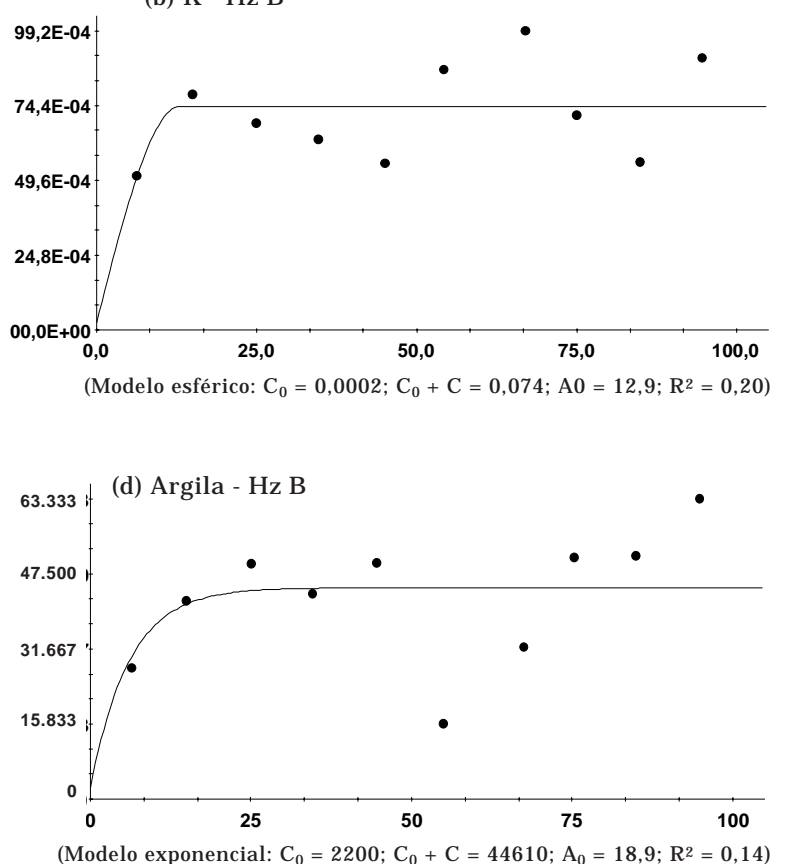

DISTÂNCIA, km

Figura 3. Variogramas do teor de potássio trocável (a e b) e do teor de argila (c e d) nos horizonte A e B. 
Quadro 2. Parâmetros dos variogramas para teor de potássio trocável e para o teor de argila nos horizontes A e B

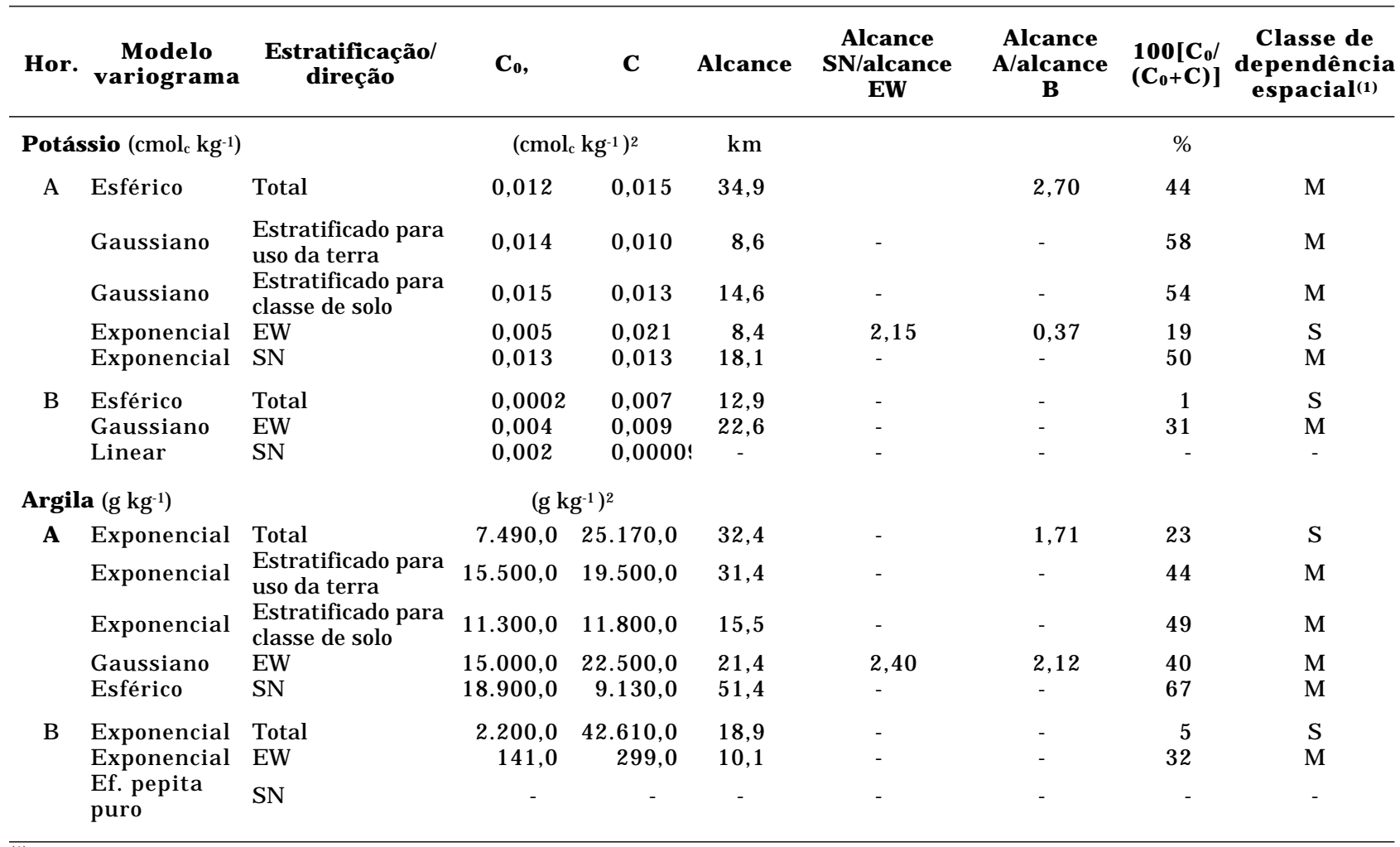

(1) S = forte dependência espacial (\% efeito pepita <25); $\mathrm{M}=$ moderada dependência espacial ( $25<\%$ efeito pepita < 75); $\mathrm{W}=$ fraca dependência espacial (\% efeito pepita > 75); R = aleatória (Cambardella et al., 1994).

(Trangmar et al., 1985). Para o horizonte B, a presença do modelo linear para o $K_{t}$, e de efeito pepita puro para o $A_{t}$, não permitiu identificar a presença de dependência espacial.

Considerando a mesma direção para o $K_{t}$, o horizonte $\mathrm{A}$, além de apresentar maior contribuição al eatória introduzida pel o maior efeito pepita que o horizonte B (Quadro 2), também mostrou maior declividade dentro das direções definidas, caracterizando grandeanisotropia (Webster, 1985), revelando que as práticas de manejo, afetando principalmente o horizonte $A$, condicionaram diferentes comportamentos na estrutura espacial da propriedade. $\mathrm{O}$ teor de argila $\left(\mathrm{A}_{t}\right)$ apresentou comportamento semel hante ao do $\mathrm{K}_{\mathrm{t}}$, neste caso, os fatores de formação do sol o têm papel preponderante na estrutura espacial daquela propriedade. Consi derando que os valores do al cance relativos aos variogramas têm uma importância considerável na determinação do limite da dependência espacial, o que pode ser também um indicativo do intervalo entre unidades de mapeamento de solos distintas (Webster, 1985), os resultados mostram que, para o horizonte A, o al cance foi de 18,1 e51,4 km no sentido sul-norte, respectivamente, para o $\mathrm{K}_{t}$ e para $A_{t}$, e de 8,4 e 21,4 km no sentido leste-oeste, respectivamente, para o $K_{t}$ e para $A_{t}$. No horizonte $B$, a presença de grande variabilidade a curta distância permitiu estimar o al cance apenas na direção leste-oeste, cujo valor foi de 22,6 km, para o $\mathrm{K}_{\mathrm{t},}$, ede $10,1 \mathrm{~km}$, para $A_{\mathrm{t}}$.

\section{Estimativas ao longo de diferentes transectos}

Para visualizar os efeitos das diferentes formas de estratificação associadas às classes de solos, o potássio trocável $\left(K_{t}\right)$ e o teor de argila $\left(A_{t}\right)$ do horizonte $A$ foram estimados ao longo dos transectos $\mathrm{T}_{1}$ e $\mathrm{T}_{2}$ em intervalos de $1,0 \mathrm{~km}$ (Figura 4). O transecto $T_{1}$ foi traçado na direção norte-sul, e o transecto $T_{2}$ na direçãoleste-oeste.

A figura 4 mostra o estimador de krigagem $\hat{\mathrm{K}}_{t}$ e $\hat{A}_{t}$, e os desvio-padrões $s_{k}$ para $T_{1}$ e $T_{2}$. Os interval os entre $\left[\left(\hat{K}_{t}+s_{k}\right)_{-}\left(\hat{K}_{t}-s_{k}\right)\right]$ e $\left[\left(\hat{A}_{t}+s_{k}\right)-\right.$ $\left(\hat{A}_{t}-S_{k}\right)$ ] são uma aproximação de $68 \%$ do intervalo de confiança para as estimativas consideradas (Cressie, 1993). As transições abruptas representadas na figura 4 correspondem às mudanças nas classes de solos. 
Os menores valores estimados na direção sulnorte (T1) foram de $0,06 \mathrm{cmol}_{\mathrm{c}} \mathrm{kg}^{-1}$, para $\hat{\mathrm{K}}_{\mathrm{t}}$, e $10,00 \mathrm{~g} \mathrm{~kg}^{-1}$, para $\hat{A}_{\mathrm{t}}$, e estavam relacionados respectivamente com a classe das Areias Quartzosas (AQa3), cerca de $130 \mathrm{~km}$ a partir do início do transecto, e a dos Latossolos Vermelho-Amarelos, cerca de $147 \mathrm{~km}$ a partir do início do transecto ( $L$ Vd8). Os maiores valores estimados foram de $0,33 \mathrm{cmol}_{\mathrm{c}} \mathrm{kg}^{-1}$, para $\hat{\mathrm{K}}_{\mathrm{t}}$, e $363,86 \mathrm{~g} \mathrm{~kg}^{-1}$ para $\hat{\mathrm{A}}_{\mathrm{t}}$, respectivamente, a 80 e $40 \mathrm{~km}$ do início do transecto, e estavam relacionados com a classe dos Podzól icos Vermelho-Amarelos eutróficos (PE 12 e PE13), os sol os mais férteis dentro da área de estudos.

As larguras máximas do intervalo de confiança, que representam a máxima amplitude de variação, foram de 0,36 $\mathrm{cmol}_{\mathrm{C}} \mathrm{kg}^{-1}$, para $\hat{\mathrm{K}}_{\mathrm{t}}$ e de $372,77 \mathrm{~g} \mathrm{~kg}^{-1}$, para $\hat{A}_{\text {, }}$ e ocorreram, respectivamente, nas unidades de mapeamento PE 8 e PE 12, correspondendo a uma distância de 90 e $80 \mathrm{~km}$ a partir do início do transecto. As larguras mínimas dos intervalos de confiança, que representam a mínima amplitude de variação para as propriedades, foram de
$0,25 \mathrm{cmol}_{\mathrm{c}} \mathrm{kg}^{-1}$, para $\hat{\mathrm{K}}_{\mathrm{t}}$, e de $220,26 \mathrm{~g} \mathrm{~kg}^{-1}$, para $\hat{A}_{\mathrm{t}}$, ocorrendo, respectivamentea 131 (AQa3) ea $69 \mathrm{~km}$ (PE 13), a partir do início do transecto.

Nosentido leste-oeste $\left(T_{2}\right)$, os maiores val ores (de $0,38 \mathrm{cmol}_{\mathrm{c}} \mathrm{kg}^{-1}$, para $\hat{\mathrm{K}}_{\mathrm{t}}$, e de $384,58 \mathrm{~g} \mathrm{~kg}^{-1}$, para $\left.\hat{A}_{\mathrm{t}}\right)$, ocorreram, respectivamente, a 61(PE 12) e $5 \mathrm{~km}$ (PE9), a partir do início do transecto. Os menores valores estimados para as duas propriedades (de $0,33 \mathrm{cmol}_{\mathrm{c}} \mathrm{kg}^{-1}$, para $\hat{\mathrm{K}}_{\mathrm{t}}$, e $188,07 \mathrm{~g} \mathrm{~kg}^{-1}$, para $\hat{A}_{\mathrm{t}}$ ) ocorreram, respectivamente, a 9 e $31 \mathrm{~km}$ a partir do início de $T_{2}$, ambas na unidade de mapeamento PE 12. As máximas amplitudes de variação para ambas as propriedades ocorreram no primeiro quilômetro do transecto estudado (T2) na uni dade de mapeamento PE 9 e corresponderam a 0,38 $\mathrm{cmol}_{\mathrm{C}} \mathrm{kg}^{-1}$, para $\hat{\mathrm{K}}_{\mathrm{t}}$, e $384,58 \mathrm{~g} \mathrm{~kg}^{-1}$, para Ât. As mínimas amplitudes de variação foram de $0,34 \mathrm{cmol}_{c} \mathrm{~kg}^{-1}$, para $\hat{\mathrm{K}}_{\mathrm{t}}$, e $188,07 \mathrm{~g} \mathrm{~kg}^{-1}$, para Ât, representando a mínima amplitude de variação das propriedades, eocorreram a 45 e a $59 \mathrm{~km}$ a partir do inicio do transecto, respectivamente, nas unidades de mapeamento PE 13 e PE 12.
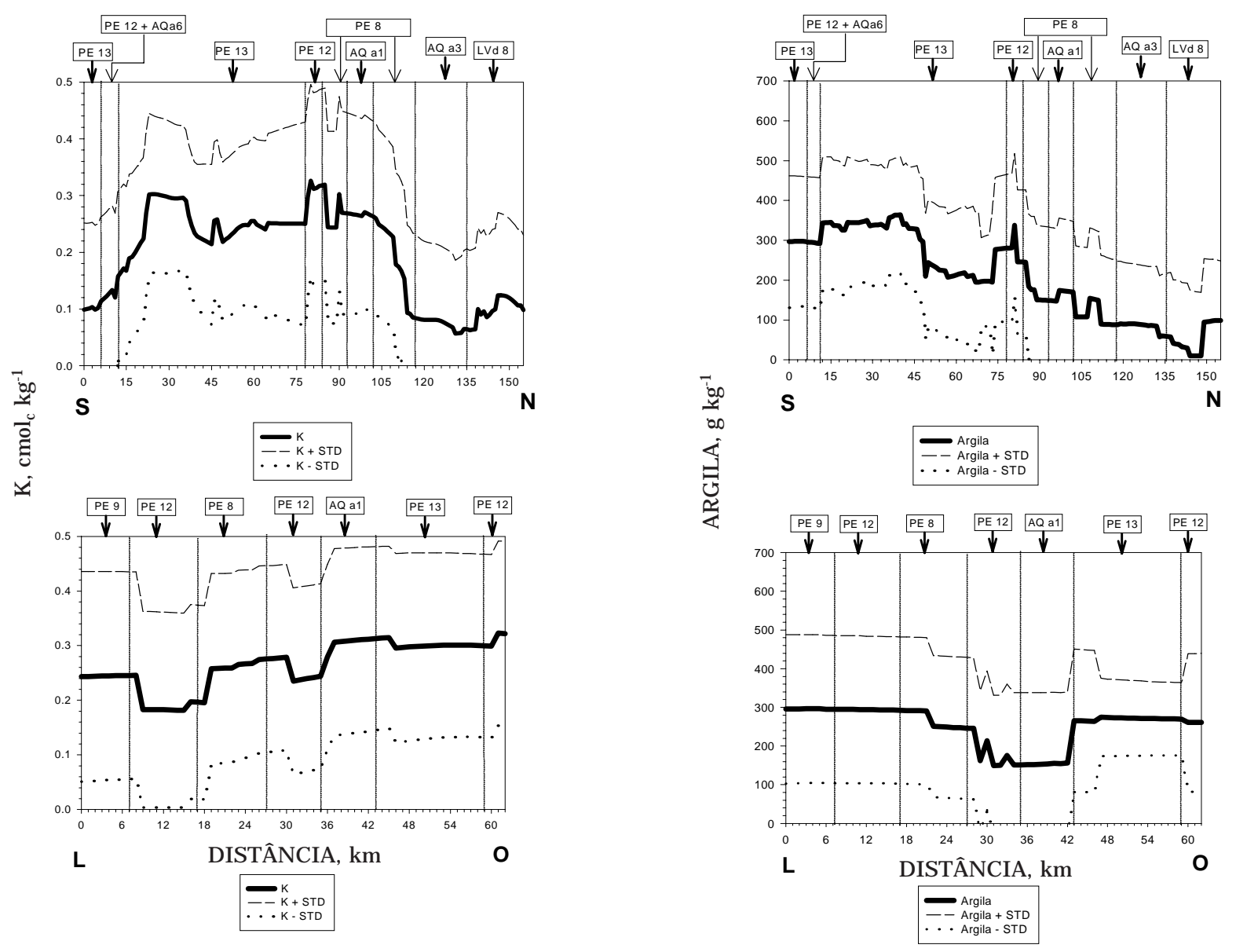

Figura 4. Valores estimados por krigagem ordinária, do teor de potássio trocável $\left(K_{t}\right)$ e do teor de argila $\left(A_{t}\right)$ ao longo dos transectos imagi nários 1 e $2\left(T_{1}\right.$ e $\left.T_{2}\right)$, para o horizonte $A$. 
As variações dos fatores de formação dos sol os ao longo dos transectos propiciaram maior variabilidade para o teor de argila do que para o potássio, o que pode ser expresso pela magnitude da amplitude total das variáveis. Para o potássio, as razões entre os valores máximos e mínimos estimados no sentido sul-norte (T1) eleste-oeste $\left(T_{2}\right)$ para o horizonte A, foram de 5,5 e 1,8 vezes, val ores bem inferiores aos encontrados para oteor de argila (36,4 e 2,0 vezes). Dessa forma, o teor de argila mostrou-se um estimador que refletiu melhor a variabilidade entre as unidades de mapeamento, o que vai ao encontro com a idéia de se usar a textura dos sol os como atributo diagnóstico para separar as classes de sol os (Oliveira et al., 1992).

\section{O uso de variogramas intra-estratos para otimizar futuras amostragens}

A partir do comportamento espacial das propriedades estudadas, avaliou-se o número de amostras necessárias para estimar o potássio trocável $\left(K_{t}\right)$ e oteor de argila $\left(A_{t}\right)$ para determinada precisão. O quadro 3 mostra a distância de amostragem $(\mathrm{km})$ e o número de amostras necessárias para estimar $K_{t}$ e $A_{t}$ com precisão de $0,15 \mathrm{cmol}_{\mathrm{C}} \mathrm{kg}^{-1}$ e $150 \mathrm{~g} \mathrm{~kg}^{-1}$, respectivamente, usando os parâmetros dos variogramas não estratificados e intra-estratos para uso da terra e classe de solo, em dois diferentes tipos de grade (quadrada e triangular).

Para o $K_{t}$, a amostragem que permitiu al cançar a precisão determinada (de $0,15 \mathrm{cmol}_{\mathrm{c}} \mathrm{kg}^{-1}$ ) com o menor número de amostras foi obtida pelo semivariograma sem estratificação com uso de uma grade triangular, o que correspondeu a um total de 29 amostras. Para esta mesma grade, a estratificação para a classe de solo mostrou uma diferença substancial quando comparada com o uso da terra, correspondendo a aproximadamente $32 \%$ menos amostras. Para $A_{t}$, a estratificação para a classe de sol o com uso de uma grade triangular proporcionou a maior eficiência, o que correspondeu apenas a 11 amostras. Neste caso, a estratificação foi extremamenteútil, reduzindo o número de amostras em $85 \%$.

\section{CONCLUSÕES}

1. O teor de potássio trocável no horizonte A na área amostrada foi mais influenciado pelas diferentes classes de solos do que pelos diferentes usos da terra e pelas práticas de manejo adotadas.

2. Os teores de potássio trocável e de argila mostraram moderada dependência espacial e revel aram anisotropia tanto nos horizontes alterados por atividades antrópicas quanto nos não alterados.

3. Nas unidades de mapeamento PE 9 ePE 12 , que apresentaram maior variabilidade (expressa por meio das larguras máximas dos intervalos de confiança), as futuras amostragens devem ser realizadas após estratificá-las em subáreas, dentro das quais a variação possa ser assumida como homogênea. Nesteestudo, o uso dos coeficientes dos semivariogramas, associados às classes de solos, proporcionou grande redução do número de amostras, considerando a precisão obtida em mapas de krigagem.

\section{AGADECIMENTOS}

Ao editor assistente, pelo trabalho criterioso de revisão que melhorou em muito o manuscrito apresentado pel os autores.

Quadro 3. Distâncias de amostragem ( $\mathrm{km}$ ) e número de amostras adicionais na área de estudos (9.802,7 km²) para estimar, respectivamente, o potássio trocável e o conteúdo de argila com precisões de $0,15 \mathrm{cmol}_{\mathrm{c}} \mathrm{kg}^{-1} \mathrm{e} 150 \mathrm{~g} \mathrm{~kg}^{-1}$, em diferentes estratificações e tipos de grade

\begin{tabular}{|c|c|c|c|c|}
\hline \multirow{2}{*}{ E stratificação } & \multicolumn{2}{|c|}{ Grade quadrada } & \multicolumn{2}{|c|}{ Grade triangular } \\
\hline & Distância & Número de amostra & Distância & Número de amostra \\
\hline Potássio $\left(\mathrm{cmol}_{\mathrm{c} \mathrm{kg}} \mathrm{kg}^{-1}\right)$ & $\mathrm{km}$ & & $\mathrm{km}$ & \\
\hline $\begin{array}{l}\text { Sem estratificação } \\
\text { Solo } \\
\text { U so da terra }\end{array}$ & $\begin{array}{l}18,70 \\
15,55 \\
12,08\end{array}$ & $\begin{array}{l}39 \\
54 \\
84\end{array}$ & $\begin{array}{l}21,43 \\
17,28 \\
13,82\end{array}$ & $\begin{array}{l}29 \\
42 \\
62\end{array}$ \\
\hline \multicolumn{5}{|l|}{ Argila $\left(\mathrm{g} \mathrm{kg}^{-1}\right)$} \\
\hline $\begin{array}{l}\text { Sem estratificação } \\
\text { Solo } \\
\text { Uso da terra }\end{array}$ & $\begin{array}{l}11,72 \\
31,25 \\
13,28\end{array}$ & $\begin{array}{l}89 \\
17 \\
71\end{array}$ & $\begin{array}{l}12,50 \\
37,50 \\
14,35\end{array}$ & $\begin{array}{l}75 \\
11 \\
58\end{array}$ \\
\hline
\end{tabular}




\section{LITERATURA CITADA}

AGUIAR, M.V.A.; COUTO, E.G. \& GUTBERLET, J . Espaçofísico da Bacia do Alto Rio Paraguai. In: SPELLER, P. \& KOHLEEP, G., eds. Estrutura sócio-econômica e dinâmica dos impactos ambientais na bacia do Alto Rio Paraguai. Cuiabá - Tübingen, UFMT/UniversidadedeTübingen. 1994. p.1-94.

BOUMA, J .; J ONGMANS, A.G.; STEIN, A. \& PEEK, G. Characterizing spatially variable hydraulic properties of a boulder clay deposit in the Netherlands. Geoderma, 45:1929, 1989

CAMBARDELLA, C.A.; MOORMAN, T.B.; NOVACK, J.M.; PARKIN, T.B.; KARLEN, D.L.; TURCO, R.F. \& KNOPKA, A. E. Field-scale variability of soil properties in central I owa soils. Soil Sci. Soc. Am. J ., 58:1240-1248, 1994.

CAMPELO J r., J.H.; CASEIRO, F.T. \& HERBSTER, O.F. Zoneamento do potencial de grãos em Mato Grosso. Cuiabá, Universidade Federal do Mato Grosso, 1990. 48p.

COUTINHO, L.M. O cerrado ea ecologia do fogo. Ci. Hoje, 12:2231, 1990.

COUTO, E.G. Variabilidade Espacial de Propriedades do solo I nfluenciadas pela Agricultura em Escala Regional eLocal, no Sul do Estado de Mato Grosso. Porto Alegre, Universidade Federal do Rio Grande do Sul, 1997. 178p. (Tese de Doutorado)

COUTO, E.G.; HIGA, N.T.; FILIPALDI , B.; AYRES, R.M. \& LOBO, F.A. O uso da terra e o garimpo na bacia do rio São L ourenço, Mato Grosso: reflexos no ambiente. Cuiabá, Universidade Federal do Mato Grosso, 1990. 206p.

COUTO, E.G.; STEIN, A. \& KLAMT, E. Large area spatial variability of soil chemical properties in central Brazil. Agric. Ecosyst. Environ., 66:139-152, 1997.

CRESSIE, N. A. C. Statistics for spatial data. New York, J ohn \& Wiley, 1993. 899p.

EMPRESA BRASILEIRA DE PESQUISA AGROPECUÁRIA EMBRAPA. Serviço Nacional de Levantamento de Solos. Manual de métodos de análise de solo. 2.ed. Rio deJ aneiro, 1997. 212p.

FROMM, H.; WINTER, K.; FILSER, J .; HANTSCHEL, R. \& $B E E S E, F$. The influence of soil type and cultivation system on the spatial distribution of the soil fauna and microorganisms and their interactions. Geoderma , 60:109118, 1993.

GOEDERT, W.J . Management of the Cerrado soils of Brazil: a review. J . Soil Sci., 34:405-428, 1983.

LOPES, A.S. \& COX, F.R. A survey of the fertility status of surface soils under "Cerrado vegetation" in Brazil. Soil Sci. Am. J ., 41:742-747, 1977.

MOGLEN, G.E. \& BRAS, R.L. The importance of spatially heterogeneous erosivity and the cumulative area distribution within a basin evolution model. Geomorphology, 12:173-185, 1995.

OLIVEIRA, J .B.; J ACOMINE, P.K.T. \& CAMARGO, M.N. Classes gerais de solos do Brasil: guia auxiliar para seu reconhecimento. J aboticabal, FUNEP, 1992. 201p.
PERRIER, E.R. \& WILDING, L. An evaluation of computational methods for field uniformity studies. Adv. Agron., 39:265312,1986

RADAMBRASIL. Levantamento de Recursos Naturais - Folha SE .21 Corumbá e parte da Fol ha SE . 20-Volume 27. Rio de J aneiro, MMESG, 1982a. 440p.

RADAMBRASIL. Levantamento de Recursos Naturais-Fol ha SD.21- Cuiabá- Volume 26. Rio deJ aneiro, MMESG, 1982b. 531p.

REICHARDT, K.; VIEIRA, S.R. \& LIBARDI, P.L. Variabilidade espacial de solos e experimentação de campo. R. Bras. Ci. Solo, 10:1-6, 1986

ROBERTSON, G.P. GS+: Geostatistics for the environment sciences - GS+ User's Guide. Plainwell, Gamma Design Software, 1998. 152p.

SCHLESINGER, W.H.; RAIKES, J .A.; HARTLEY, A.E. \& CROSS, A.F. On the spatial pattern of soil nutrients in desert ecosystems. Ecology, 77: 364-375, 1996.

SELBY, M. J . Hillslope materials and processes. 2. ed. Oxford, Oxford University Press, 1993. 451p.

SOUZA, L.D.S.; COGO, N.P. \& VIEIRA, S.R. Variabilidade de propriedades físicas equímicas do solo em um pomar cítrico. R. Bras. Ci. Solo, 21:367-372, 1997.

SPSS. SPPSS for windows base system user's guide release 6.0. Chicago, 1994. 828p.

STEIN, A. OPTIM PC software. Wageningen. Wageningen Agricultural University, 1996.

STEIN, A. \& STARITSKY, I.G. Manual for the geostatistical programs SPATANAL, CROSS and MAPIT. Wageningen, Wageningen Agricultural University, 1993. 19p.

STEIN, A. Spatial statistics for soils and the environment Preliminary version. Enschede, ITC, 1998. 47p. (Lecture Notes)

TRANGMAR, B.B.; YOST, R.S. \& UEHARA, G. Applications of geostatistics to spatial studies of soil properties. Adv. Agr., 38:45-94,1985.

TRIMBLE. General reference, GPS pathfinder system. Sunnyvale, Trimble Navigation, 1993. não paginado.

UEHARA, G.; TRANGMAR, B.B. \& YOST, R.S. Spatial variability of soil properties. In: Workshop of the ISSS and the SSSA. Las Vegas, 1984. Proceedings. Wageningen, Pudoc, 1985. p.61-95.

UPCHURCH, D.R. \& EDMONDS, W.J . Statistical procedures for specific objectives. Madison, Soil Science Society of American, 1991. p.49-71 (SSSA Special Publication, 28)

van der BERG, M. \& KLAMT, E. Variabilidade espacial de características de sol os da região do Planalto Médio, RS. R. Bras. Ci. Solo, 21:393-399, 1997.

VOLTZ, M.; LAGACHERIE, P. \& LOUCHART, X. Predicting soil properties over a region using sample information from a mapped reference area. Europ. J . Soil Sci., 48:19-30, 1997.

WEBSTER, R. Quantitative spatial analysis of soil in the field. In: STEWART, B.A., ed. Advance in soil science, vol.3. New York, Spriger-Verlag, 1985. p.1-70. 
WI LKINSON, L. Systat for Windows. Evanston, Systat I nc., 1992. 547p.

WINKELS, H.J.\& STEIN, A. Optimal cost-effective sampling for monitoring and dredging of contaminated sediments. J . Environ. Qual., 26:933-946, 1997.
YOST, R.S.; UEHARA, G. \& FOX, R.L. Geostatistical analysis of soil chemical properties of large areas. I. Semi-variograms. Soil Sci Soc. Am. J ., 46:1028-1032, 1982a.

YOST, R.S.; UEHARA, G. \& FOX, R.L. Geostatistical analysis of soil chemical properties of large areas. II. Kriging. Soil Sci Soc. Am. J ., 46:1033-1037, 1982b. 